\title{
NÍVEL DE ATIVIDADE FÍSICA E CORRELAÇÃO COM VALORES GLICÊMICOS DE CARDIOPATAS PARTICIPANTES DE UM PROJETO DE EXTENSÃO
}

Laís Manata Vanzella, Beatriz Espanhol Garcia, Ana Paula Soares dos Santos, Elaine Aparecida de Oliveira, Carolina Takahashi, Thais Cabral Almeida, Paula Rapchan dos Santos, Aline Ferreira Lima Gonçalves, Ana Alice Soares dos Santos, Rayana Loch Gomes, Aline Fernanda Barbosa Bernardo, Luiz Carlos Marques Vanderlei

Universidade Estadual Paulista Júlio de Mesquita Filho - UNESP. E-mail: lais.vanzella@ig.com.br

\section{RESUMO}

Doenças cardiovasculares (DCV) são importantes causas de morte, influenciada por fatores de riscos (FR) como diabetes mellitus (DM) e sedentarismo, que são potencializados quando associados. O objetivo do trabalho foi avaliar o nível de atividade física (AF) correlacionado ao índice glicêmico de participantes do setor de reabilitação cardíaca do Centro de Estudos e Atendimento em Fisioterapia e Reabilitação (CEAFiR). Para isso, incluiu-se indivíduos que apresentavam algum tipo de cardiopatia ou FR para DCV e que estavam em tratamento fisioterapêutico, e aplicado um questionário de atividade física (BAECKE). Classificam-se como sedentários $80 \%$ na atividade física ocupacional, 50\% na atividade física de locomoção, 45\% para atividade física de tempo livre e atividade física total, porém não há significância quando correlacionados com índices glicêmicos. Conclui-se que apesar de não haver correlação entre os índices avaliados, o sedentarismo é um FR para DCV e para a DM, sendo extremamente importante a pratica de AF.

Palavras-chave: doenças cardiovasculares, fatores de risco, diabetes mellitus, sedentarismo, prevenção.

\section{PHYSICAL ACTIVITY LEVEL AND CORRELATION WITH GLYCEMIC VALUES IN CARDIAC INDIVIDUALS PARTICIPANTS OF AN EXTENSION PROJECT}

\begin{abstract}
Cardiovascular diseases are important causes of death, influenced by risk factors like diabetes mellitus and sedentary, that when in association can be potentiated. However, the object of this work is to evaluate the physical activity level and the glycemic index in participants of extension project. For this, we include individuals with glucose $>100 \mathrm{mg} / \mathrm{dl}$, verified by the finger prick method, and applied a physical activity questionnaire (BAECKE). Therefore, in the classification of the subjects we found that $80 \%$ are sedentary in work index, $50 \%$ in sport index, and $45 \%$ at leisure-time index and total physical activity, but we did not find significance when we compare this with glycemic index. We conclude that we did not find correlation between the evaluated indexes, but the sedentary is a risk factor for cardiovascular disease, and for diabetes mellitus, so is too important the physical activity practice.
\end{abstract}

Keywords: cardiovascular disease, risk factors, diabetes mellitus, sedentary, prevention. 


\section{INTRODUÇÃO}

As doenças cardiovasculares (DCV) são as principais causas de morte no Brasil, correspondendo a $30 \%$ dos óbitos em diversas faixas etárias, além de representarem as principais causas de permanência hospitalar prolongada e serem responsáveis pela principal alocação de recursos públicos em hospitalizações ${ }^{1}$. Essas doenças são influenciadas, em sua maioria, por um conjunto de fatores de risco (FR), sendo alguns considerados não modificáveis, como a hereditariedade, sexo e idade, e outros modificáveis, mediante alterações no estilo de vida, como a hipertensão arterial (HA), diabetes mellitus (DM), dislipidemia, obesidade, sedentarismo, estresse e tabagismo ${ }^{2}$ A associação de comportamentos não saudáveis com fatores de risco (FR) apresenta uma maior predisposição dos indivíduos a desenvolver $\mathrm{DCV}^{3}$.

A DM se destaca por ser um dos maiores FR independente para $D^{4} V^{4}$. Ela se caracteriza por hiperglicemia resultante de defeitos na secreção da insulina, ação da insulina ou ambos, que acarretam anomalias no metabolismo de carboidratos, gorduras e proteínas ${ }^{5}$. Nas últimas décadas, a DM tem se tornado um sério e crescente problema de saúde pública nos países desenvolvidos e em desenvolvimento, devido ao aumento de sua prevalência, morbidade e mortalidade ${ }^{6}$.
Acredita-se que este aumento se deve ao crescimento e envelhecimento populacional, à maior urbanização e à crescente prevalência de obesidade e sedentarismo, bem como à maior sobrevida do paciente com $\mathrm{DM}^{7}$. Os países em desenvolvimento se destacam pela maior incidência, com crescente proporção de pessoas afetadas em grupos etários mais jovens ${ }^{7}$. No Brasil, no final da década de 1980 estimou-se a prevalência de DM na população adulta em $7,6 \%$, e, dados mais recentes apontam para taxas mais elevadas com aumento de $2,7 \%$ na faixa etária de 30 a 59 anos e de 17,4\% na faixa de 60 a 69 anos, ou seja, um aumento de 6,4 vezes $^{7}$.

$\mathrm{Na}$ tentativa de diminuir os valores glicêmicos e a incidência da DM, a prática de atividade física $(\mathrm{AF})$ tem sido determinante. Uma vez, que o sedentarismo promove aumento desses valores e ainda, está associado à obesidade, aumentando o risco cardiovascular ${ }^{8}$. Além disso, estudos têm demonstrado que a progressão do estado de tolerância diminuída à glicose pode ser inibida, ou pelo menos adiada por mudanças em hábitos alimentares e/ou pelo aumento da pratica de $\mathrm{AF}^{9}$. Em meta analise realizada em 14 estudos, exercícios resultaram em uma redução clínica significante na $\mathrm{Hba}_{1 c}$ em pacientes com diabetes tipo $2^{10}$. Pacientes do tipo 1 apresentam características de resistência periférica à insulina, que pode 
também, ser melhorada por meio da $\mathrm{AF}^{11}$, formando a base de uma teoria ao qual o exercício também pode influenciar no controle glicêmico de diabetes tipo 1. Assim, podemos observar a importância de se obter informações sobre o nível de AF da população, em seus mais variados aspectos.

Cardiopatas que frequentam programas de reabilitação cardíaca foram considerados sedentários para as diversas categorias de $\mathrm{AF}$ e a incidência de $\mathrm{FR}$, nessa população, também foi elevada ${ }^{3}$. Porém, apesar de haver a associação da DM com o sedentarismo, nada se afirma sobre a influência da falta de $A F$, em diferentes categorias, sobre os valores glicêmicos. Visto a potencialização da agregação dessas variáveis para agravamento e desenvolvimento da DCV, é importante investigar a influência do sedentarismo em diferentes categorias sobre os valores glicêmicos de indivíduos cardiopatas. O objetivo do estudo consiste em caracterizar as categorias de AF de cardiopatas participantes de um projeto de extensão e frequentadores do setor de Reabilitação Cardíaca do Centro de Estudos e Atendimento em Fisioterapia e Reabilitação (CEAFiR) e correlacionar o nível de AF em cada categoria com os valores glicêmicos desses indivíduos.

\section{MATERIAIS E MÉTODOS}

Para realização desse estudo foram recrutados indivíduos idosos de ambos os sexos, participantes de um projeto de extensão intitulado: Projeto de prevenção primária e secundária de doenças cardiovasculares, e frequentadores do Centro de Estudos e Atendimento em Fisioterapia e Reabilitação CEAFIR da FCT/UNESP Presidente Prudente. Foram incluídos os voluntários que apresentavam algum tipo de cardiopatia ou FR para DCV, e que estavam em tratamento fisioterapêutico. Foram excluídos do estudo voluntários que não apresentaram condições cognitivas para responder as perguntas realizadas, ou que se recusaram a realizar as coletas.

Assim, foram selecionados 20 voluntários, sendo 12 homens e 08 mulheres. Os procedimentos deste estudo foram aprovados pelo Comitê de Ética em Pesquisa da Instituição (CEP: 2013/331954) e todos os voluntários foram devidamente informados sobre os procedimentos e objetivos do estudo, e após concordarem, assinaram um termo de consentimento livre e esclarecido.

\section{Protocolo experimental}

O protocolo experimental foi composto por duas etapas. A Primeira consistiu em avaliação inicial com o objetivo de obter informações quanto à identificação e anamnese dos voluntários e a mensuração 
da glicemia. Na segunda etapa, foi aplicado na forma de entrevista individual por profissionais treinados o inventário de Baecke $^{12}$ para a determinação das categorias de AF.

\section{Avaliação Inicial}

Os voluntários foram identificados quanto ao nome, idade, sexo e se possui diagnóstico de DCV e/ou DM. A taxa de glicose foi verificada pelo exame de punção de polpa digital através do capilar, utilizandose 0 aparelho OneTouch (Johnson, Argentina), todos no período da manhã, em jejum de 12 horas.

\section{Avaliação do nível de atividade física}

Para avaliação das diferentes categorias de AF foi aplicado o questionário de AF preconizada por BAECKE $^{12}$, em forma de entrevista, onde os voluntários foram orientados a questionar sobre qualquer dúvida que surgisse.

O questionário consiste em 16 itens contendo 4 diferentes componentes: ocupacional, locomoção, esporte e tempo livre. Todas as respostas são divididas em 5 pontos de escala com exceção do nome da ocupação e o tipo de esporte praticado. As questões são distribuídas em 3 seções distintas: 1) atividade física ocupacional (AFO); 2) atividade física de tempo livre
(AFTL) e 3) atividade física de locomoção $(\mathrm{AFL})$.

A AFO é avaliada por meio das questões 1 a 8 , levando em consideração as atividades realizadas durante $\mathrm{o}$ período ocupacional, distinguindo o tipo de ocupação ${ }^{13}$, o tempo permanecido em pé e a quantidade de peso carregada pelos indivíduos durante o trabalho ${ }^{12,14}$.

A investigação da AFTL foi feita pela avaliação da pratica de exercícios físicos regulares (questão 9) envolvendo modalidades específicas. Com base na intensidade (leve, moderada e vigorosa) ${ }^{13}$, frequência e duração da atividade foi calculado um escore específico para essa questão ${ }^{12,14}$. O escore engloba mais três questões (10 a 12) que comparam as atividades físicas de tempo livre com pessoas da mesma idade, a presença de suor e a pratica de esportes sem regularidade. Lembrando que todos os voluntários são frequentadores de um programa de reabilitação cardíaca, todos os pacientes obtiveram o mesmo escore na questão 9, exceto aqueles que praticavam regularmente algum esporte, além da reabilitação cardiovascular.

Para avaliação da AFL, as questões referem-se às atividades de assistir à televisão (atividade sedentária), caminhar, andar de bicicleta, e, uma ultima questão, sobre os minutos por dia em atividades de 
locomoção (caminhar ou uso de bicicleta para ir e voltar do trabalho, escola ou compras) ${ }^{12,14}$.

Foram classificados como sedentários os indivíduos localizados abaixo da média do escore da população em cada categoria de AF.

\section{Análise estatística}

A estatística descritiva foi realizada para caracterização da amostra estudada e os resultados foram apresentados com valores de média, desvio padrão, números absolutos e relativos.

Para a correlação dos dados, foi testada inicialmente a normalidade pelo teste de Shapiro-wilk e em seguida foi aplicada a correlação de Pearson para aqueles que foram considerados dados normais ou a correlação de Spearman para aqueles classificados como não normais. $A$ magnitude da correlação foi considerada fraca para $r$ de 0 a 0,40; moderada para $r$ de 0,41 a 0,80 e forte para $r \geq 0,81$. Considerada significante $p<0,05$.

\section{RESULTADOS}

Foram avaliados 20 voluntários, que apresentaram mulheres com média de $66,80 \pm 6,41$ anos, homens com $64,18 \pm 9,78$ anos e $112,75 \pm 37,58 \mathrm{mg} / \mathrm{dl}$ para glicemia de jejum.

Na tabela 1 encontra-se a prevalência de sedentarismo de acordo com os resultados obtidos na aplicação do inventário de Baecke. Nota-se que a maioria dos voluntários foram considerados sedentário apenas para a categoria de AFO.

Tabela 1. Distribuição dos pacientes em relação ao nível de atividade física em cada categoria

\begin{tabular}{ccc}
\hline Domínio & Sedentário & Ativo \\
\hline AFO & $0 \pm 0$ & $2,59 \pm 0,52$ \\
& $16(80 \%)$ & $4(20 \%)$ \\
AFTL & $2,58 \pm 0,38$ & $3,63 \pm 0,37$ \\
& $9(45 \%)$ & $11(65 \%)$ \\
AFL & $1,63 \pm 0,41$ & $2,90 \pm 0,50$ \\
& $10(50 \%)$ & $10(50 \%)$ \\
AFT & $4,88 \pm 0,67$ & $7,00 \pm 0,78$ \\
& $9(45 \%)$ & $11(65 \%)$
\end{tabular}

Média \pm desvio padrão. AFO = atividade física ocupacional; AFTL = atividade física de tempo livre; $A F L=$ atividade física de locomoção; AFT = atividade física total.

Na tabela 2 observa-se a correlação entre os níveis glicêmicos da população 
avaliada e os diferentes escores encontrados em cada classificação do Baecke. Nota-se que não houve significância em nenhuma as correlações abaixo.

Tabela 2. Correlação entre a taxa de glicemia e os domínios do questionário Baecke.

\begin{tabular}{ccccc}
\hline Variável & AFO & AFTL & AFL & AFT \\
\hline \multirow{2}{*}{ Glicemia } & $\boldsymbol{r}$ & $\boldsymbol{r}$ & $\boldsymbol{r}$ & $\boldsymbol{r}$ \\
& 0,03660 & 0,005311 & $-0,1525$ & $-0,09718$ \\
& $\boldsymbol{p}$ & $\boldsymbol{p}$ & $\boldsymbol{p}$ & $\boldsymbol{p}$ \\
& 0,8783 & 0,9823 & 0,5209 & 0,6836 \\
\hline
\end{tabular}

Normalidade dos dados (Shapiro-wilks); dados normais (correlação de spearman). AFO = atividade física ocupacional; $\mathrm{AFTL}=$ atividade física de tempo livre; $\mathrm{AFL}$ = atividade física de locomoção; $\mathrm{AFT}$ = atividade física total.

\section{DISCUSSÃO}

Os principais achados mostram que o maior número de indivíduos classificados como sedentários pelo inventário de Baecke foi na AFO, representando $80 \%$ destes seguidos da AFL, AFTL E AFT. Ainda, observou-se que não houve correlação quando comparado o índice glicêmico destes indivíduos com as diferentes categorias do Baecke.

No que diz respeito à $A F O$, ao qual obtivemos $80 \%$ dos indivíduos avaliados, justifica-se pelo fato de a maioria dos indivíduos serem aposentados. Quanto à AFTL, e AFT, ambas apresentaram os mesmos valores de porcentagem, $45 \%$. O programa de reabilitação ao qual estes indivíduos apresentam foi incluído na questão ${ }^{15}$ como um esporte, na categoria AFTL. Porém, a questão não era determinante para que o indivíduo fosse considerado ativo, visto que outras questões englobavam esta categoria e influenciaram na classificação da mesma.

Na categoria AFL, 10 dos indivíduos avaliados foram considerados sedentários, ou seja $50 \%$ da população. Acredita-se que este resultado foi encontrado por se tratar de uma população em sua maioria aposentada, e que não praticam demais atividades além da reabilitação cardiovascular.

A pratica de AF é considerada uma variável complexa de ser analisada, com particularidades em diferentes domínios que precisam ser consideradas, como, AF no trabalho, em casa e durante os horários de lazer, que são indicadores de saúde física e mental $^{16}$.

Sabemos como descrito acima, que a inatividade física é responsável por um elevado número de mortes a nível mundial, inclusive pela ocorrência de diabetes do tipo $2^{17}$, porém, quando correlacionado o 
sedentarismo nos diferentes domínios do BAECKE, e a DM, não foram encontradas diferenças significantes nestes índices, podendo ser justificado pelo reduzido tamanho amostral utilizado, ou até por características especificas da população incluída no estudo, que frequenta um programa de reabilitação regularmente e possuem cuidados extras por já apresentarem uma patologia.

Observamos que apesar de não ser encontrado diferenças significantes em nosso estudo, o grupo Finn Diane Study ${ }^{18}$ à observou quando correlacionou glicemia com a pratica de AF em mulheres. Além disso, sabemos os efeitos benéficos da pratica de AF em indivíduos diagnosticados com DM e DCV. Portanto, o trabalho vem enfatizar a importância da reabilitação cardiovascular nas condições de saúde acima apresentadas.

Pode-se citar como limitações do estudo a dificuldade em adequar aos critérios utilizados para a classificação do Baecke, os programas de reabilitação cardiovascular ao qual estes indivíduos frequentam, sendo este incluído na questão relacionada ao esporte por conseguir determinar a frequência com que esta atividade é realizada.
A partir dos resultados podemos concluir que a maioria dos indivíduos são sedentários para a categoria de AFO e os valores de glicêmicos estão acima dos valores de normalidade. Ainda, não há correlação entre valores glicêmicos e nível de AF.

\section{REFERENCIAS}

1. Coltro RS, Mizutani BM, Mutti A, Délia MPB, Martinelli LMB, et al. Frequência de fatores de risco cardiovasculares em voluntários participantes de evento de educação em saúde. Ver Assoc Med Bras. 2009;55(5):606-10.

DOI:

http://dx.doi.org/10.1590/S0104$\underline{42302009000500028}$

2. Pansani AP, Anequini IP, Vanderlei $\mathrm{LCM}$, Tarumoto $\mathrm{MH}$. Prevalência de fatores de risco para doenças coronarianas em idosos frequentadores de um programa "Universidade Aberta à Terceira Idade". ArqCiênc Saúde. 2005;12(1):27-31.

3. Bernardo AFB, Rossi RC, Souza NM, Pastre CM, Vanderlei LCM. Associação entre atividade física e fatores de risco cardiovasculares em indivíduos de um programa de reabilitação física. Rev Bras Med Esporte. 2013;19(4):231-5. DOI: http://dx.doi.org/10.1590/S1517$\underline{86922013000400001}$

4. Gus I, Fischmann A, Medina C. Prevalência dos Fatores de Risco da Doença Arterial Coronariana no Estado do Rio Grande do Sul. ArqBrasCardiol. 2002;78(5):478-83. http://dx.doi.org/10.1590/S0066-

782X2002000500005

5. American Diabetes Association. Diagnosis and Classification of Diabetes Mellitus. Diabetes Care. 2010;33(1). DOI: http://dx.doi.org/10 2337/dc10-S062.

\section{CONCLUSÃO}


6. Sociedade Brasileira de Diabetes. Diretrizes da Sociedade Brasileira de Diabetes. 2009.

7. Sociedade Brasileira de Diabetes. Diretrizes da Sociedade Brasileira de Diabetes. 2013-2014.

8. Tavares TB, Nunes SM, Santos MO. Obesidade e qualidade de vida: Revisão da literatura. Ver Med Minas Gerais 2010;20(3):359-366.

9. DPP Research Group: Reduction in the incidence of type 2 diabetes with lifestyle intervention or metformin. N Engl J Med. 2002;346:393-403. DOI: http://dx.doi.org/10 1056/NEJMoa012512.

10. Boule NG, Haddad E, Kenny GP, WellsGA, Sigal RJ. Effects of exercise on glycemic control and body mass intype 2 diabetes mellitus: a metaanalysis of controlled clinical trials.2001;286(10):121827. DOI: http://dx.doi.org/10 1001/jama.286.10.1218.

11. Arslanian SA, Suprasongsin C. Differences in the vivo insulin secretion and sensitivity of healthy black versus white adolescents. J Pediatr. 1996;129:440-3. DOI: http://dx.doi.org/10 1016/S00223476(96)70078-1.

12. Baecke JA, Burema J, Frijters JE. A short questionnaire for the measurement of habitual physical activity in epidemiological studies. Am J Clin Nutr 1982;36:936-42.

13. Farinatti PTV. Apresentação de uma Versão em Português do Compêndio de Atividades Físicas: uma contribuição aos pesquisadores e profissionais em Fisiologia do Exercício. Revista Brasileira de Fisiologia do Exercício 2003;3:177-208.

14. Florindo AA, Latorre MRDO. Validação e reprodutibilidade do questionário de Baecke de avaliação da atividade física habitual em homens adultos Revista Brasileira de Medicina do Esporte 2003;9(3):121-128.

15. Godoy MF, Takakura IT, Correa PR. Comportamento dinâmico não-linear (Teoria do Caos) como elemento prognóstico de morbidade e mortalidade em pacientes submetidos à cirurgia de revascularização miocardia. Arq Ciênc Saúde 2005;12(4)16771.

16. Freitas IF, Rodrigues JEQ, Sousa S, Campos EZ, Fernandes RA, et al. Relacionamento de diferentes domínios da atividade física habitual com indicadores de risco cardiovascular em adultos jovens do sexo masculino.2010;16(3):591-597. DOI: http://dx.doi.org/10 $5016 / 1980$ 6574.2010v16n3p591.

17. Souza CA, César CLG, Barros MBA, Carandina $\mathrm{L}$, Goldbaum $\mathrm{M}$, et al. Prevalência de atividade física no lazer e fatores associados: estudo de base populacional em são Paulo, Brasil. Cad. Saúde Pública. 2013;29(2):270-282. DOI: http://dx.doi.org/10 1590/S0102311X201300020001.

18. Wadén, J.; Tikkanen, H.; Forsblom, C.;Fagerudd, J.; Pettersson-Fernholm, K.; Lakka, T.; Riska, M.; Groop, P.H.;Finndiane Study Group. Leisure time physical activity is associated with poor glycemic control in type 1 diabetic women: the FinnDiane study group. Diabetes Care 2005; 28 (4): 777-82. Diabetes Care. 2005;28(4):777-782. DOI: http://dx.doi.org/10 2337/diacare.28.4.777

Recebido para publicação em 17/08/2014 Revisado em 01/09/2014

Aceito em 04/09/2014 